\section{REDUCE NEEDLESTICK INJURY RISK}

A quick and simple way to reduce the risk of needlestick injuries is to use InSafe Safety syringes from Initial Medical Services - a safety system providing comprehensive protection for clinical staff from the beginning of the procedure through to the disposal of the needle.

InSafe's syringe and sharps box ensure that the contaminated needle is never exposed except during the actual injection. It feels and aspirates just like a traditional syringe so there will be no interruptions to the dental practice when introducing the protective system. When the injection has been administered, the protective sleeve locks securely into place over the needle, protecting clinical staff and patients when not in use.

The needle can then be safely disposed of using Initial Medical's specially designed sharps container.

Aspirating and non-aspirating syringes are available; they are compatible with all standard dental needles and cartridges. The 1 litre sharps container is ideal for all dental locations and has a secure base that ensures the container can never be knocked over.

On 11 May the new Health Et Safety (Sharps Instruments in Healthcare) Regulations 2013 comes into force, translated into national law from the European sharps injuries directive. Under the directive, practices need to assess the risk of exposure to blood-borne infections from sharps injuries, identify how to eliminate this and where exposure cannot be eliminated, put into place extensive prevention methods.

For more information on InSafe Safety syringes and sharps disposal boxes visit www.initial.co.uk/medical-services/dental-waste/index.html.

\title{
DELIVERING WARM GUTTA-PERCHA THROUGHOUT THE CANAL
}

Delivering warm gutta-percha throughout the canal, GuttaCore is a gutta-percha obturator from DENTSPLY which makes prepar-

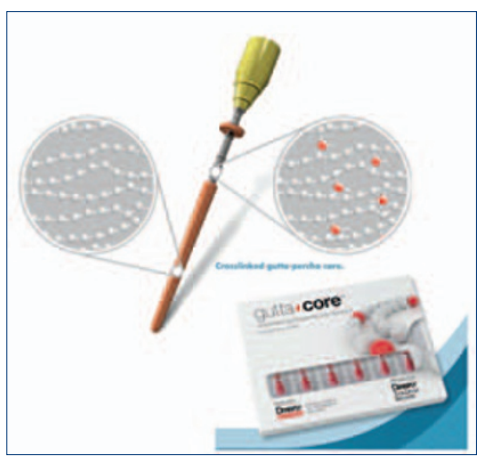

ing post space and retreatment easier when compared with plastic carriers.

Designed to create a dense, 3D fill that follows the curves of the canal quickly, GuttaCore has a core made of crosslinked gutta-percha. This makes it the perfect filling material for centrally condensing the surrounding flowable gutta-percha into the root canal system.

As with any DENTSPLY product, user simplicity is of paramount importance and GuttaCore is no different. The handle of the GuttaCore is made so that it can be removed easily by bending it from side to side without impacting upon the seal.

Call 0800072 3313; access webinars and product demonstrations and earn CPD at dentsplyacademy. co.uk.

\section{PAVING THE WAY TO PRACTICE SUCCESS}

Find your ideal partnership or bespoke coaching service with 7connections, the brand new business that paves the way for dental practices to achieve success. The 7connections team has an unparalleled understanding of the business of dentistry and offers a tailor made service to suit the unique needs of every practice.

Guiding you every step of the way, 7connections offers:

1. Training - showing your team how to do things

2. Consultancy - helping your team to apply the training in practice

3. Coaching - holding you and your team accountable for progress

4. Management - helping you to implement systems

5. Leadership - teaching you to lead your team

6. Mentoring - sharing our experience

7. Partnership - releasing equity and joining you in the ownership of your practice.

Find the missing link to your dream practice with 7connections, for better dentistry and first class patient care. For more information about 7connections call Chris Barrow on 07713 644437 or visit www.coachbarrow.com/tag/7connections/.

\section{ULTRA-FAST, POWDER-FREE SCANNING}

Planmeca PlanScan is a digital impression scanner for ultra-fast, powder-free 3D scanning. The quick and accurate scanner provides real-time digital impressions from one-tooth to full-arch scans. The open STL data output means that the scanned data can be sent to any dental lab for CAD work. Planmeca PlanScan is the first dental unit integrated impression scanner. Alternatively, it is available as a standalone version and can be connected eg to a laptop.

Planmeca also offers dentists a new open CAD software suite for easy 3D design. Planmeca PlanCAD Easy is integrated in the Planmeca Romexis software and is a perfect tool for designing prosthetic works from individual inlays to full-arch bridges and abutments.

The ready design can then be sent to Planmeca PlanMill 40, a new milling unit targeted for dentists and designed for glass ceramic and other material works. The 4-axis milling unit

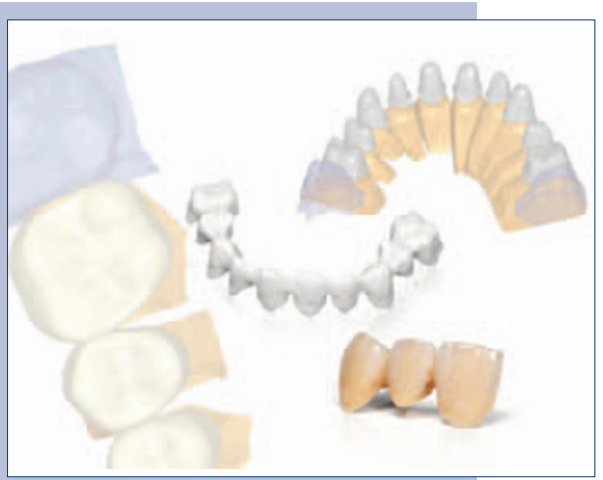

is quick and accurate. www.planmeca.com 